\title{
A Posteriori Improvement of Cubic Spline Approximate Solution of Two-Point Boundary Value Problem
}

By

\author{
Manabu SAKAI*
}

\begin{abstract}
We consider the numerical solution of two-point boundary value problem by collocation method using cubic spline. We derive asymptotic expansions of the errors which are a posteriori determined with little additional computation. The applications of these asymtotic expansions to a posteriori improvement of the approximate solution and adaptive mesh selection strategy (chopping procedure) are discussed. Some numerical results which closely correspond with the predicted theory are given.
\end{abstract}

\section{§1. Introduction and Description of Method}

Cubic splines are of much use for approximating solutions of twopoint boundary value problems for both linear and nonlinear ordinary differential equations. In the present paper we shall consider the following two-point boundary value problem:

$$
x^{\prime \prime}(t)=f\left(t, x(t), x^{\prime}(t)\right), \quad 0 \leq t \leq 1
$$

with boundary conditions

$$
\begin{aligned}
& a_{0} x(0)-b_{0} x^{\prime}(0)=c_{0} \\
& a_{1} x(1)+b_{1} x^{\prime}(1)=c_{1}
\end{aligned}
$$

where $f(t, x, w)$ is defined and sufficiently smooth in a region $D$ of $(t, x, w)$-space intercepted by two hyper-planes $t=0$ and $t=1$.

We rewrite the above problem in the following form:

$$
\begin{array}{ll}
x^{\prime}(t)=w(t), & 0 \leq t \leq 1 \\
w^{\prime}(t)=f(t, x(t), w(i)), & 0 \leq t \leq 1
\end{array}
$$

Communicated by S. Hitotumatu, January 31, 1983.

* Department of Mathematics, Faculty of Science, Kagoshima University, Kagoshima 890, Japan. 


$$
\begin{aligned}
& a_{0} x(0)-b_{0} w(0)=c_{0} \\
& a_{1} x(1)+b_{1} w(1)=c_{1} .
\end{aligned}
$$

Now making use of B-spline $Q_{m+1}(t)$ :

$Q_{m+1}(t)=(1 / m !) \sum_{i=0}^{m+1}(-1)^{i}\left(\begin{array}{c}m+1 \\ i\end{array}\right)(x-i)_{+}^{m}$, we consider spline functions of the form $x_{h}(t)=\sum_{i=-3}^{n-1} \alpha_{i} Q_{4}(t / h-i)$ and $w_{h}(t)=\sum_{i=-2}^{n-1} \beta_{i} Q_{3}(t / h-i)$ $(n h=1)$ with undetermined coefficients $\alpha_{-3}, \alpha_{-2}, \cdots, \alpha_{n-1}$ and $\beta_{-2}$, $\beta_{-1}, \cdots, \beta_{n-1}$. The above $x_{h}$ and $w_{h}$ will be approximate solutions to the problem (1.4)-(1.7) if they satisfy

$$
\begin{array}{ll}
x_{h}^{\prime}(t)=w_{h}(t), & 0 \leq t \leq 1 \\
w_{h}^{\prime}(t)=P f\left(t, x_{h}(t), w_{h}(t)\right), & 0 \leq t \leq 1 \\
a_{0} x_{h}(0)-b_{0} w_{h}(0)=c_{0} & \\
a_{1} x_{h}(1)+b_{1} w_{h}(1)=c_{1} . & \\
{ }_{2} . &
\end{array}
$$

Here $P$ is an operator defined by

$$
(P g)(t)=\sum_{i=0}^{n} g\left(t_{i}\right) L_{i}(t)
$$

where $L_{i}(t)$ is a piecewise linear function with property $L_{i}\left(t_{j}\right)=\delta_{i, j}$ $\left(t_{j}=j h\right)$. From a well known relation $Q_{m+1}^{\prime}(t)=Q_{m}(t)-Q_{m}(t-1)$, we see that equation (1.8) is equivalent to the following system of $n+2$ equations:

$$
(1 / h)\left(\alpha_{i}-\alpha_{i-1}\right)=\beta_{i}, i=-2,-1, \cdots, n-1 .
$$

Any two piecewise linear functions coincide with each other if and only if they coincide at the nodes, therefore we see that (1.9) is equivalent to the following system of $n+1$ equations:

$$
\begin{aligned}
& (1 / h)\left(\beta_{i-1}-\beta_{i-2}\right)=f\left(t_{i},(1 / 6)\left(\alpha_{i-1}+4 \alpha_{i-2}\right.\right. \\
& \left.\left.\quad+\alpha_{i-3}\right), \frac{1}{2}\left(\beta_{i-1}+\beta_{i-2}\right)\right), i=0,1, \cdots, n .
\end{aligned}
$$

The boundary conditions $(1.10)-(1.11)$ give two equations:

$$
\begin{aligned}
& \left(a_{0} / 6\right)\left(\alpha_{-1}+4 \alpha_{-2}+\alpha_{-3}\right)-\left(b_{0} / 2\right)\left(\beta_{-1}+\beta_{-2}\right)=c_{0} \\
& \left(a_{1} / 6\right)\left(\alpha_{n-1}+4 \alpha_{n-2}+\alpha_{n-3}\right)+\left(b_{1} / 2\right)\left(\beta_{n-1}+\beta_{n-2}\right)=c_{1} .
\end{aligned}
$$

The number of undetermined coefficients is $2 n+5$ and the conditions (1.12)-(1.15) precisely give the requisite number of equations. In practical computations it is more convenient to use the equations containing only $\alpha_{i}(i=-3,-2, \cdots, n-1)$ which can be obtained from (1.12)-(1.15) by eliminating $\beta_{i}(i=-2,-1, \cdots, n-1)$. 
In the present paper we assume that the problem (1.4)-(1.7) has an isolated solution $(\hat{x}(t), \hat{w}(t))$ satisfying the internality condition

$$
\begin{gathered}
U=\{(t, x, w)|| x-\hat{x}(t)|+| w-r \hat{v}(t) \mid \leq \delta, \\
t \in[0,1]\} \subset D
\end{gathered}
$$

for some $\delta>0$. The solution $(\hat{x}(t), \hat{w}(t))$ to the problem (1.4)-(1.7) is isolated if and only if

$$
G=\left[\begin{array}{ll}
a_{0} & -b_{0} \\
a_{1} y_{1}(1)+b_{1} z_{1}(1) & a_{1} y_{2}(1)+b_{1} z_{2}(1)
\end{array}\right]
$$

is nonsingular, where

$$
\left[\begin{array}{ll}
y_{1}(t) & y_{2}(t) \\
z_{1}(t) & z_{2}(t)
\end{array}\right]=\Phi(t)
$$

is a fundamental matrix with property $\Phi(0)=E$ ( $E$ the unit matrix) of the first variation equation of $(1.4)-(1.5)$ with respect to $(\hat{x}(t)$, $\hat{w}(t))$, that is,

$$
\begin{aligned}
& y^{\prime}(t)=z(t) \\
& z^{\prime}(t)=f_{x}(t, \hat{x}(t), \hat{w}(t)) y(t)+f_{w}(t, \hat{x}(t), \hat{w}(t)) z(t) .
\end{aligned}
$$

Then we have

Theorem 1 ([10]). In a sufficiently small neighbourhood of the isolated solution $(\hat{x}(t), \hat{w}(t))$ of the problem (1.4)-(1.7), there is a spline function $\left(\bar{x}_{h}(t), \bar{w}_{h}(t)\right)$ of the form

$$
\begin{aligned}
& \bar{x}_{h}(t)=\sum_{i=-3}^{n-1} \bar{\alpha}_{i} Q_{4}(t / h-i) \\
& \bar{w}_{h}(t)=\sum_{i=-2}^{n-1} \bar{\beta}_{i} Q_{3}(t / h-i)
\end{aligned}
$$

such that

$$
\begin{aligned}
& || \hat{x}-\bar{x}_{h}|!=\max | \hat{x}(t)-\bar{x}_{h}(t) \mid=O\left(h^{2}\right) \\
& \left\|\hat{w}-\bar{w}_{h}\right\|=O\left(h^{2}\right) .
\end{aligned}
$$

On using this Theorem 1, we shall prove the following asymptotic expansions of the errors $\hat{x}(t)-\bar{x}_{h}(t)$ and $\hat{w}(t)-\bar{w}_{h}(t)$.

Theorem 2. If $(\hat{x}, \hat{w})$ is the isolated solution of the problem (1.4)-(1.7), then we have

$$
\hat{x}(t)-\bar{x}_{h}(t)=-\left(h^{2} / 12\right) \phi(t)+O\left(h^{4}\right)
$$




$$
\hat{w}(t)-\bar{w}_{h}(t)=-\left(h^{2} / 12\right) \phi(t)+O\left(h^{3}\right)
$$

where $(\phi, \phi)$ is the solution of the following differential equation:

$$
\begin{array}{ll}
(1.23) & \phi^{\prime}(t)=\phi(t) \\
(1.24) & \phi^{\prime}(t)=f_{x}(t, \hat{x}(t), \hat{w}(t)) \phi(t)+f_{w}(t, \hat{x}(t), \hat{w}(t)) \phi(t)+\hat{x}^{(4)}(t) \\
(1.25) & a_{0} \phi(0)-b_{0} \phi(0)=0 \\
(1.26) & a_{1} \phi(1)+b_{1} \phi(1)=0 .
\end{array}
$$

Remark. At mesh points, we have (1. 27) $\hat{x}^{\prime \prime}(t)-\bar{x}_{h}^{\prime \prime}(t)=-\left(h^{2} / 12\right)\left\{\phi^{\prime \prime}(t)-\hat{x}^{(4)}(t)\right\}+O\left(h^{4}\right), h \rightarrow 0$.

From the above Remark, we have $O\left(h^{2}\right)$-approximations to $\hat{x}_{i}^{(4)}$, $i=0,1, \cdots, n$ :

$$
\left\{\begin{aligned}
\hat{x}_{0}^{(4)}= & 1 /\left(12 h^{2}\right)\left\{35 \bar{x}_{h}^{\prime \prime}\left(t_{0}\right)-104 \bar{x}_{h}^{\prime \prime}\left(t_{1}\right)\right. \\
& \left.+114 \bar{x}_{h}^{\prime \prime}\left(t_{2}\right)-56 \bar{x}_{h}^{\prime \prime}\left(t_{3}\right)+11 \bar{x}_{h}^{\prime \prime}\left(t_{4}\right)\right\}+O\left(h^{2}\right) \\
\hat{x}_{i}^{(4)}= & 1 / h^{2}\left\{\bar{x}_{h}^{\prime \prime}\left(t_{i+1}\right)-2 \bar{x}_{h}^{\prime \prime}\left(t_{i}\right)+\bar{x}_{h}^{\prime \prime}\left(t_{i-1}\right)\right\} \\
& +O\left(h^{2}\right), i=1,2, \cdots, n-1 \\
\hat{x}_{n}^{(4)}= & 1 /\left(12 h^{2}\right)\left\{35 \bar{x}_{h}^{\prime \prime}\left(t_{n}\right)-104 \bar{x}_{h}^{\prime \prime}\left(t_{n-1}\right)\right. \\
& \left.+114 \bar{x}_{h}^{\prime \prime}\left(t_{n-2}\right)-56 \bar{x}_{h}^{\prime \prime}\left(t_{n-3}\right)+11 \bar{x}_{h}^{\prime \prime}\left(t_{n-4}\right)\right\}+O\left(h^{2}\right)
\end{aligned}\right.
$$

where for any function $g(t)$ let us denote $g\left(t_{i}\right)$ by $g_{i}$.

Here we consider an approximate problem to (1.23)-(1.26):

$$
\begin{aligned}
\phi_{h}^{\prime}(t)= & \phi_{h}(t) \\
\phi_{h}^{\prime}(t)= & f_{x}\left(t, \bar{x}_{h}(t), \bar{w}_{h}(t)\right) \phi_{h}(t) \\
& \quad+f_{w}\left(t, \bar{x}_{h}(t), \bar{w}_{h}(t)\right) \phi_{h}(t)+g_{h}(t) \\
a_{0} \phi_{h}(0) & -b_{0} \phi_{h}(0)=0 \\
a_{1} \phi_{h}(1) & +b_{1} \phi_{h}(1)=0
\end{aligned}
$$

where $g_{h}(t)$ is a piecewise linear function and $g_{h}\left(t_{i}\right)$ are the righthand sides of (1.28) without $O\left(h^{2}\right)$.

Since $G$ is nonsingular, i. e., the solution of the problem (1.23)(1.26) exists, in virtue of (1.19)-(1.20) the problem $(1.23)^{\prime}-(1.26)^{\prime}$ has a solution $\left(\hat{\phi}_{h}, \hat{\phi}_{h}\right)$ for sufficiently small $h$. By $(1.28)$, we have

$$
\left\|\hat{x}^{(4)}-g_{h}\right\|=O\left(h^{2}\right)
$$

from which follow

$$
\left\|\phi-\hat{\phi}_{h}\right\|_{1},\left\|\phi-\hat{\phi}_{h}\right\|=O\left(h^{2}\right) .
$$


Now we consider the numerical solution of the problem $(1.23)^{\prime}-$ $(1.26)^{\prime}$ by using the operator $P$ :

$$
\begin{aligned}
& \bar{\phi}_{h}^{\prime}(t)= \bar{\phi}_{h}(t) \\
& \bar{\phi}_{h}^{\prime}(t)= P\left[f_{x}\left(t, \bar{x}_{h}(t), \bar{w}_{h}(t)\right) \bar{\phi}_{h}(t)\right. \\
&\left.\quad+f_{w}\left(t, \bar{x}_{h}(t), \bar{\omega}_{h}(t)\right) \bar{\phi}_{h}(t)+g_{h}(t)\right] \\
& a_{0} \bar{\phi}_{h}(0)-b_{0} \bar{\phi}_{h}(0)=0 \\
& a_{1} \bar{\phi}_{h}(1)+b_{1} \bar{\phi}_{h}(1)=0 .
\end{aligned}
$$

Since the solution $\left(\hat{\phi}_{h}, \hat{\phi}_{h}\right)$ cf the problem $(1.23)^{\prime}-(1.26)^{\prime}$ is isolated, the approximate problem $(1.31)-(1.34)$ has a solution $\left(\bar{\phi}_{h}\right.$, $\left.\bar{\phi}_{h}\right)$ such that

$$
\left\|\bar{\phi}_{h}-\hat{\phi}_{h}\right\|, \quad \mid \bar{\phi}_{h}-\hat{\phi}_{h} \|=O\left(h^{2}\right)
$$

for sufficiently small $h$.

Combining (1.30) and (1.35) yields

$$
\left\|\phi-\bar{\phi}_{h}\right\|,\left\|\phi-\bar{\phi}_{h}\right\|=O\left(h^{2}\right)
$$

from which follow computable asymptotic expansions:

$$
\begin{aligned}
& \hat{x}(t)-\bar{x}_{h}(t)=-\left(h^{2} / 12\right) \bar{\phi}_{h}(t)+O\left(h^{4}\right) \\
& \hat{w}(t)-\bar{w}_{h}(t)=-\left(h^{2} / 12\right) \bar{\phi}_{h}(t)+O\left(h^{3}\right) .
\end{aligned}
$$

Since the coefficient matrix of the linear system (1.31)-(1.34) for determining $\left(\bar{\phi}_{h}, \bar{\phi}_{h}\right)$ is the one of the Newton method at the final stage by which we calculate $\left(\bar{x}_{h}, \bar{w}_{h}\right)$, the principal parts of the errors of (1.37)-(1.38) are obtained with little additional computation.

In Section 3, we consider chopping procedure applied to two-point boundary value problem by Russell and Christansen ([8]). Our chopping procedure uses only uniform meshes at each step which can be automatically refined in order to reduce the (estimated) error below a requested tolerance. It behaves quite adequately for many problems including ones whose solutions have sharp gradients. We see that our procedure performs efficiently and reliably on all the problems considered, within the limitations imposed by the maximum number of grid points allowed. That limitation is computer dependent. We emphasize that our packages presented later have provisions for estimating and controlling the global error of the computed solution with little additional computation. 


\section{§2. Asymptotic Expansions of Errors}

Let $e_{1}=\hat{x}-\bar{x}_{h}$ and $e_{2}=\hat{w}-\bar{w}_{h}$. By Theorem 1 we have

$$
\begin{aligned}
e_{1}^{\prime}(t)= & e_{2}(t) \\
e_{2}^{\prime}(t)= & f_{2}(t) e_{1}(t)+f_{3}(t) e_{2}(t) \\
& +(I-P) \hat{x}^{\prime \prime}(t)+R(t)+O\left(h^{4}\right) \\
a_{0} e_{1}(0) & -b_{0} e_{2}(0)=0 \\
a_{1} e_{1}(1) & +b_{1} e_{2}(1)=0
\end{aligned}
$$

where

$$
\begin{aligned}
& f_{2}(t)=f_{x}(t, \hat{x}(t), \hat{w}(t)), f_{3}(t)=f_{w}(t, \hat{x}(t), \hat{w}(t)) \\
& R(t)=-(I-P)\left(f_{2} e_{1}+f_{3} e_{2}\right) \quad(I \text { the unit operator). }
\end{aligned}
$$

Since $\left\|e_{1}\right\|,\left\|e_{2}\right\|=O\left(h^{2}\right), e_{1}^{\prime}(t)=e_{2}(t)$ and $e_{1}^{\prime \prime}(t)=e_{2}^{\prime}(t)$, in virtue of the well-known inequality:

$$
\left\|g^{\prime}\right\| \leq c_{0}\|g\|+c_{1}\left\|g^{\prime}\right\| \text { for any } g \in C^{2}[0,1],
$$

we have

$$
\|R\| \leq O\left(h^{2}\right)\left\|\left(f_{2} e_{1}+f_{3} e_{2}\right)^{\prime \prime}\right\| \leq O\left(h^{4}\right)+O\left(h^{2}\right)\left\|e_{2}^{\prime \prime}\right\| .
$$

By a simple calculation, we have

$$
\begin{aligned}
e_{2}^{\prime \prime}(t)= & \hat{w}^{\prime \prime}(t)-\tau \bar{w}_{h}^{\prime \prime}(t)=\hat{w}^{\prime \prime}(t)-(1 / h)\left\{\bar{w}_{h}^{\prime}\left(t_{i+1}\right)-\bar{w}_{h}^{\prime}\left(t_{i}\right)\right\} \\
= & (1 / h)\left\{e_{2}^{\prime}\left(t_{i+1}\right)-e_{2}^{\prime}\left(t_{i}\right)\right\}+O\left(h^{2}\right) \\
= & (1 / h)\left\{f_{2}\left(t_{i+1}\right) e_{1}\left(t_{i+1}\right)+f_{3}\left(t_{i+1}\right) e_{2}\left(t_{i+1}\right)\right. \\
& \left.-f_{2}\left(t_{i}\right) e_{1}\left(t_{i}\right)-f_{3}\left(t_{i}\right) e_{2}\left(t_{i}\right)\right\}+O\left(h^{2}\right) \\
= & f_{3}\left(t_{i}\right)\left\{e_{2}\left(t_{i+1}\right)-e_{2}\left(t_{i}\right)\right\} / h+O\left(h^{2}\right) \\
= & (1 / h) f_{3}\left(t_{i}\right) \int_{t_{i}}^{t_{i+1}} e_{2}^{\prime}(t) d t+O\left(h^{2}\right)
\end{aligned}
$$

from which follows by using again (2.2)

$$
\left\|e_{2}^{\prime \prime}\right\| \leq O\left(h^{2}\right)+C\|R\|
$$

for some constant $C$ independent of $h$.

By combining (2.5) and (2.7), we have the estimate of $\|R\|$ of the form

$$
\|R\|=O\left(h^{4}\right)
$$

for sufficiently small $h$.

Since $(\hat{x}, \hat{w})$ is isolated, by (2.1)-(2.4) we have

$$
\left[\begin{array}{l}
e_{1} \\
e_{2}
\end{array}\right]=\int_{0}^{1} H(\cdot, s)\left[\begin{array}{c}
0 \\
(I-P) \hat{x}^{\prime \prime}(s)
\end{array}\right] d s+\left[\begin{array}{l}
O\left(h^{4}\right) \\
O\left(h^{4}\right)
\end{array}\right],
$$


where

$$
H(t, s)= \begin{cases}\Phi(t)\left[E-G^{-1} A_{1} \Phi(1)\right] \Phi^{-1}(s), & s \leq t \\ -\Phi(t) G^{-1} A_{1} \Phi(1) \Phi^{-1}(s), & s>t\end{cases}
$$

$$
\mathrm{A}_{1}=\left[\begin{array}{cc}
0 & 0 \\
a_{1} & b_{1}
\end{array}\right]
$$

Since $(I-P) \hat{x}^{\prime \prime}(t)=\frac{1}{2} \hat{x}^{(4)}\left(t_{i+\frac{1}{2}}\right)\left(t-t_{i}\right)\left(t-t_{i+1}\right)+(1 / 6) \hat{x}^{(5)}\left(t_{i+\frac{1}{2}}\right)\left(t-t_{i}\right)$. $\left(t-t_{i+\frac{1}{2}}\right)\left(t-t_{i+1}\right)+O\left(h^{4}\right) \quad\left(t_{i+\frac{1}{2}}=\left(i+\frac{1}{2}\right) h\right)$, we have for $K(t, s)=$ $H_{12}(t, s)$ or $H_{22}(t, s)$

$$
\begin{aligned}
I\left(t_{j}\right)= & \int_{0}^{1} K\left(t_{j}, s\right)(I-P) \hat{x}^{\prime \prime}(s) d s \\
= & \sum_{i=0}^{n-1}\left\{\frac{1}{2} \hat{x}^{(4)}\left(t_{i+\frac{1}{2}}\right) \int_{t_{i}}^{t_{i+1}} K\left(t_{j}, s\right)\left(s-t_{i}\right)\left(s-t_{i+1}\right) d s\right. \\
& +(1 / 6) \hat{x}^{(5)}\left(t_{i+\frac{1}{2}}\right) \int_{t_{i}}^{t_{i+1}} K\left(t_{j}, s\right)\left(s-t_{i}\right) \\
& \left.\times\left(s-t_{i+\frac{1}{2}}\right)\left(s-t_{i+1}\right) d s\right\}+O\left(h^{4}\right) \\
= & -\left(h^{3} / 12\right) \sum_{i=0}^{n-1} \hat{x}^{(4)}\left(t_{i+\frac{1}{2}}\right) K\left(t_{j}, t_{i+\frac{1}{2}}\right)+O\left(h^{4}\right) \\
= & -\left(h^{2} / 12\right) \int_{0}^{1} K\left(t_{j}, s\right) \hat{x}^{(4)}(s) d s+O\left(h^{4}\right) .
\end{aligned}
$$

Hence we have

$$
\left[\begin{array}{l}
e_{1}\left(t_{j}\right) \\
e_{2}\left(t_{j}\right)
\end{array}\right]=-\left(h^{2} / 12\right) \int_{0}^{1} H\left(t_{j}, s\right)\left[\begin{array}{c}
0 \\
\hat{x}^{(4)}(s)
\end{array}\right] d s+\left[\begin{array}{l}
O\left(h^{4}\right) \\
O\left(h^{4}\right)
\end{array}\right]
$$

from which follow asymptotic expansions (1.21)-(1.22) at mesh points. Since

$$
\begin{aligned}
& \bar{x}_{h}\left(t_{j}\right)-\left(h^{2} / 12\right) \bar{\phi}_{h}\left(t_{j}\right)=\hat{x}\left(t_{j}\right)+O\left(h^{4}\right) \\
& \bar{x}_{h}^{\prime}\left(t_{j}\right)-\left(h^{2} / 12\right) \bar{\phi}_{h}^{\prime}\left(t_{j}\right)\left(=\bar{w}_{h}\left(t_{j}\right)-\left(h^{2} / 12\right) \bar{\phi}_{h}\left(t_{j}\right)\right) \\
& \quad=\hat{x}^{\prime}\left(t_{j}\right)+O\left(h^{4}\right), j=0,1, \cdots, n,
\end{aligned}
$$

$\bar{x}_{h}-\left(h^{2} / 12\right) \bar{\phi}_{h}$ is the cubic interpolatory spline to $\hat{x}$, so we have the desired asymptotic expansions (1.21)-(1.22) at any point $t \in[0,1]$.

\section{§3. Chopping Procedure}

We have frequently observed that when problems are solved on a sequence of meshes, an acceptable solution is arrived at some regions before the problem as a whole has been solved. A successful 
strategy of ours for keeping the work to a minimum while selecting successive partitions has been to chop off these regions where the solution is satisfactory. The differential equation is then solved on the remaining regions using asymptotic expansions (1.21)-(1.22) for the boundary conditions.

If $\left(h^{2} / 12\right)\left|\bar{\phi}_{h}(t)\right| \leq \varepsilon$ ( $\varepsilon$ a desired tolerance)

$$
\text { for } t \in[0, a],[b, 1]\left(a=n_{1} h, b=n_{2} h\right) \text {, }
$$

let us consider the following boundary value problem on the remaining interval $[a, b]$ :

$$
\begin{aligned}
& x^{\prime \prime}(t)=f\left(t, x(t), x^{\prime}(t)\right), \quad a \leq t \leq b \\
& x(a)=\bar{x}_{h}(a)-\left(h^{2} / 12\right) \bar{\phi}_{h}(a) \\
& x(b)=\bar{x}_{h}(b)-\left(h^{2} / 12\right) \bar{\phi}_{h}(b)
\end{aligned}
$$

where possibly we have

$$
\begin{aligned}
& \left|\hat{x}(a)-\left\{\bar{x}_{h}(a)-\left(h^{2} / 12\right) \bar{\phi}_{h}(a)\right\}\right| \ll \varepsilon \\
& \left|\hat{x}(b)-\left\{\bar{x}_{h}(b)-\left(h^{2} / 12\right) \bar{\phi}_{h}(b)\right\}\right| \ll \varepsilon .
\end{aligned}
$$

If $\left(h^{2} / 12\right)\left|\bar{\phi}_{h}(t)\right| \leq \varepsilon$ for $t \in[a, b]$, then we consider the following two problems on the remaining intervals $[0, a]$ and $[b, 1]$ :

$$
\begin{aligned}
& x^{\prime \prime}(t)=f\left(t, x(t), x^{\prime}(t)\right), \quad 0 \leq t \leq a \\
& a_{0} x(0)-b_{0} x^{\prime}(0)=c_{0} \\
& x(a)=\bar{x}_{h}(a)-\left(h^{2} / 12\right) \bar{\phi}_{h}(a) . \\
& x^{\prime \prime}(t)=f\left(t, x(t), x^{\prime}(t)\right), \quad b \leq t \leq 1 \\
& x(b)=\bar{x}_{h}(b)-\left(h^{2} / 12\right) \bar{\phi}_{h}(b) \\
& a_{1} x(1)+b_{1} x^{\prime}(1)=c_{1} .
\end{aligned}
$$

By reducing $h$ to $h / 2$ and using the operator $P$, we consider the numerical solution of problems (3.1)-(3.3), or (3.6)-(3.8) and (3.9)-(3.11). The successive use of this procedure will give the approximate solution $\bar{x}_{h}(t)$ so that

$$
\left\|\hat{x}-\left\{\bar{x}_{h}-\left(h^{2} / 12\right) \bar{\phi}_{h}\right\}\right\| \ll\left\|\hat{x}-\bar{x}_{h}\right\| \leq \varepsilon \text {. }
$$

\section{§4. Numerical Illustration}

In this section, we discuss numerical results obtained from some concrete examples. These results confirm the theoretical accuracies established in previous sections. The rate of decrease of the errors $O\left(h^{\alpha}\right)$, where $\alpha$ is computed from the results from $h=1 / 16$ to $1 / 32$, 
is given in parentheses in each Table. As our examples, we choose

\section{Problem 1.}

$$
\begin{aligned}
& x^{\prime \prime}(t)=\frac{1}{2}\left\{x^{2}(t)+x^{\prime 2}(t)\right\} / \exp (t), \\
& x(0)-x^{\prime}(0)=0 \\
& x(1)+x^{\prime}(1)=2 e .
\end{aligned}
$$

Problem 2. The same differential equation as in Problem 1 subject to the boundary conditions:

$$
x(0)=1, x(1)=e .
$$

The exact solutions of the above problems are $\exp (t)$.

In the following Tables, the left and right sides of $(\cdots) \rightarrow(\cdots)$ mean $\max _{i}\left|\hat{x}^{(k)}\left(t_{i}\right)-\bar{x}_{h}^{(k)}\left(t_{i}\right)\right|$ and $\max _{i}\left|\hat{x}^{(k)}\left(t_{i}\right)-\left\{\bar{x}_{h}^{(k)}\left(t_{i}\right)-\left(h^{2} / 12\right) \bar{\phi}_{h}^{(k)}\left(t_{i}\right)\right\}\right|, k$ $=0,1$, respectively.

Table 4.1. The observed maximum errors for function values.

\begin{tabular}{c|c|c|c}
\hline$h$ & $1 / 16$ & $1 / 32$ & $\alpha$ \\
\hline Problem 1 & $0.209-3^{*} \rightarrow 0.334-6$ & $0.523-4 \rightarrow 0.211-7$ & $2.0 \rightarrow 4.0$ \\
Problem 2 & $0.613-4 \rightarrow 0.993-7$ & $0.152-4 \rightarrow 0.620-8$ & $2.0 \rightarrow 4.0$ \\
\hline
\end{tabular}

* We denote $0.209 \times 10^{-3}$ by $0.209-3$.

Table 4.2. The observed maximum errors for derivatives.

\begin{tabular}{c|c|c|c}
\hline$h$ & $1 / 16$ & $1 / 32$ & $\alpha$ \\
\hline Problem 1 & $0.186-3 \rightarrow 0.330-6$ & $0.464-4 \rightarrow 0.208-7$ & $2.0 \rightarrow 4.0$ \\
Problem 2 & $0.344-3 \rightarrow 0.828-6$ & $0.861-4 \rightarrow 0.521-7$ & $2.0 \rightarrow 4.0$ \\
\hline
\end{tabular}

Here we may consider another definition of an operator $\bar{P}$, i.e.,

$$
(\bar{P} g)(t)=\sum_{i=0}^{n} \beta_{i} L_{i}(t)
$$

such that

$$
\left\{\begin{array}{l}
\Delta^{r} \beta_{0}=0, \quad r \geq 4 \\
\beta_{i}=g_{\imath}, i=1,2, \cdots, n-1 \\
\nabla^{r} \beta_{n}=0
\end{array}\right.
$$


where $\Delta$ and $\nabla$ are forward and backward difference operators, respectively. For this operator $\bar{P}$, we have the same results in Theorems 1 and 2. Since the above definition of $\bar{P}$ does not need function values at $t=0$ and $t=1$, by using this we may consider the numerical solution of the following singular boundary value problem:

$$
\begin{aligned}
& x^{\prime \prime}(t)+(\gamma / t) x^{\prime}(t)+f(t, x(t))=0, \quad 0<t \leq 1 \\
& x^{\prime}(0)=0, x(1)=c_{1}
\end{aligned}
$$

with $\gamma=0,1,2$.

Problem 3. Now we consider a nonlinear boundary value problem:

$$
\begin{aligned}
& x^{\prime \prime}(t)+(2 / t) x^{\prime}(t)+x^{5}(t)=0, \quad 0<t \leq 1 \\
& c_{1}=\sqrt{3} / 2 .
\end{aligned}
$$

This problem has a unique solution $1 / \sqrt{1+t^{2} / 3}$.

Problem 4. Consider another nonlinear problem:

$$
\begin{aligned}
& x^{\prime \prime}(t)+(1 / t) x^{\prime}(t)+\exp (x(t))=0, \quad 0<t \leq 1 \\
& c_{1}=0 .
\end{aligned}
$$

The solutions are $\hat{x}(t)=2 \ln \left[(B+1) /\left(B t^{2}+1\right)\right]$, where $B=3 \pm 2 \sqrt{2}$. In the following Table, we list up the numerical results for the smaller solution.

Table 4.3. The observed maximum errors for function values.

\begin{tabular}{c|c|c|c}
\hline$h$ & $1 / 16$ & $1 / 32$ & $\alpha$ \\
\hline Problem 3 & $0.216-4 \rightarrow 0.278-6$ & $0.545-5 \rightarrow 0.174-7$ & $2.0 \rightarrow 4.0$ \\
Problem 4 & $0.490-4 \rightarrow 0.168-7$ & $0.123-4 \rightarrow 0.105-8$ & $2.0 \rightarrow 4.0$ \\
\hline
\end{tabular}

Table 4.4. The observed maximum errors for derivatives.

\begin{tabular}{c|c|c|c}
\hline$h$ & $1 / 16$ & $1 / 32$ & $\alpha$ \\
\hline Problem 3 & $0.384-4 \rightarrow 0.371-6$ & $0.963-5 \rightarrow 0.230-7$ & $2.0 \rightarrow 4.0$ \\
Problem 4 & $0.675-4 \rightarrow 0.800-7$ & $0.169-4 \rightarrow 0.498-8$ & $2.0 \rightarrow 4.0$ \\
\hline
\end{tabular}

Next we consider the application of chopping procedure to the 
following problems in which we take a desired tolerance $\varepsilon=10^{-4}$ and $h=1 / 32$ as starting mesh sizes.

Problem 5. First we consider the singular perturbation problem:

$$
\begin{aligned}
& 10^{-4} x^{\prime \prime}(t)-x(t)=1, \quad 0 \leq t \leq 1 \\
& x(0)=1, x(1)=1 .
\end{aligned}
$$

There exists a unique solution symmetric about $t=\frac{1}{2}$ and having boundary layers of thickness $1 / 100$ at 0 and 1 . That is, it is troublesome for methods based on standard initial value problem techniques.

\section{Problem 6.}

$$
\begin{aligned}
& 10^{-4} x^{\prime \prime}(t)+\left(1-\frac{1}{2} t\right) x^{\prime}(t)-\frac{1}{2} x(t)=0 \\
& x(0)=0, x(1)=1 .
\end{aligned}
$$

The exact solution is characterized by a boundary layer cf thickness $10^{-4}$ at the origin, and a limiting interior solution is $1 /(2-t)$. From numerical results, we have 4999 as an approximation to $\hat{x}^{\prime}(0)$.

Problem 7. Now we consider an equation which arises in the stress distribution in a spherical membrance with normal and tangential loads:

$$
\begin{aligned}
& x^{\prime \prime}(t)+\{3 \operatorname{cotan}(t)+2 \tan (t)\} x^{\prime}(t)+0.7 x(t)=0 \\
& 30^{\circ} \leq t \leq 60^{\circ}
\end{aligned}
$$

subject to the boundary conditions

$$
x\left(30^{\circ}\right)=0, x\left(60^{\circ}\right)=5 .
$$

The solution curve has a sharp spike approximately at $30.66^{\circ}$ with the magnitude of the solution at this point approximately $283.26 \cdots$.

In the following Table, $a=30^{\circ}$ and $N$ is the maximum number cf grid points on the remaining intervals, that is, we have to solve a linear system of order $N+3$ at least one time. In Problem 5, only the half of the interval $[0,1]$ is covered because of the symmetry. 
Table 4.5. Remaining subintervals.

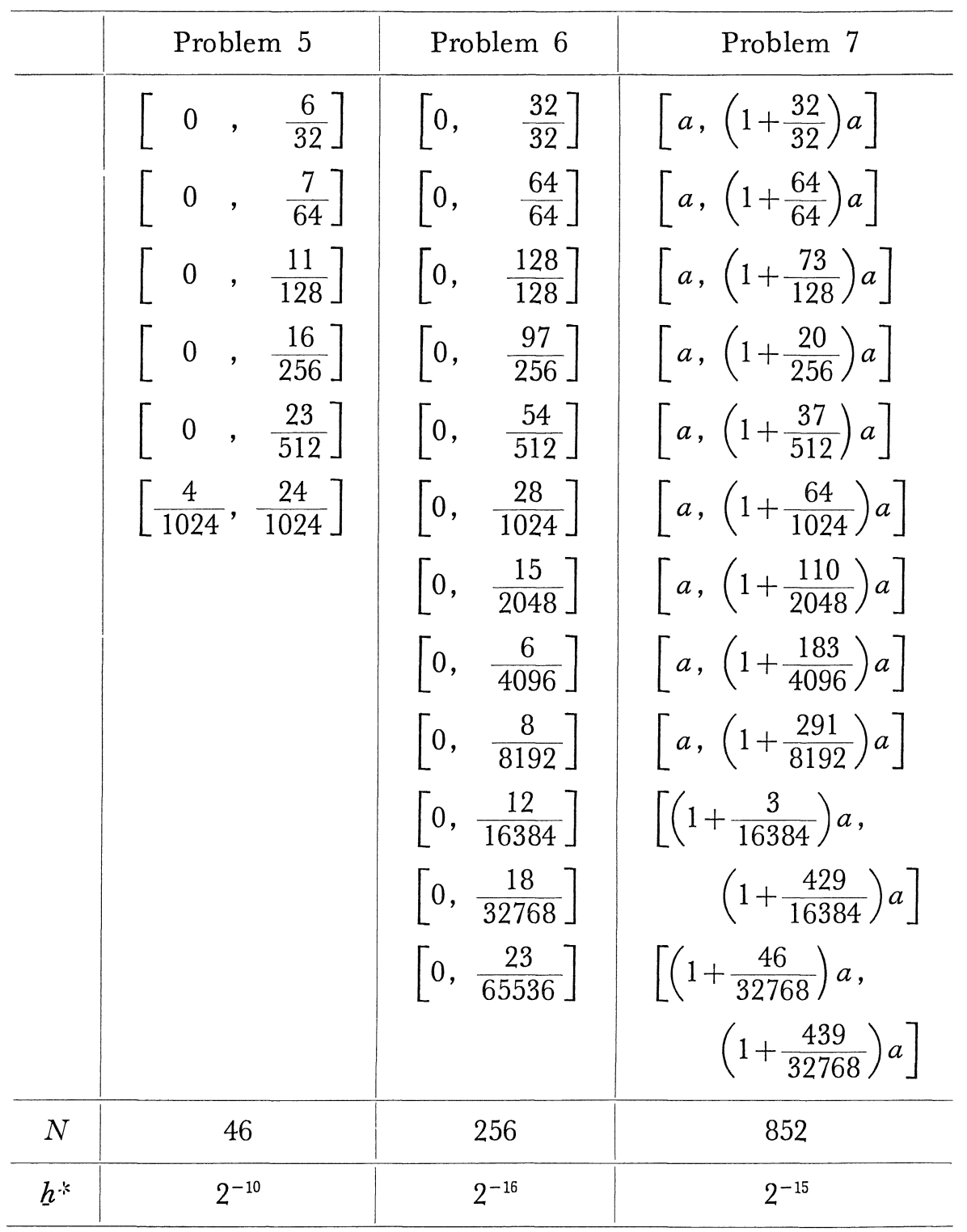

* $\underline{h}$ is the smallest mesh size of the remaining subintervals. 


\section{References}

[1] Ahlberg, J., Nilson, E. and Walsh, J., The theory of splines and their applications, Academic Press, New York, 1967.

[2] Henrici, P., Discrete variable methods in ordinary differential equations, J. Wiley \& Sons, New York, 1964.

[3] Jacobs, D., Boundary value problems in ordinary differential equations, The state of the art in numerical analysis, D. Jacobs, ed., Academic Press, New York, 1977.

[4] Kantorovich, L. and Akilov, G., Functional analysis in normed spaces, Pergamon, New York, 1964.

[5] Keller, H., Accurate difference methods for linear ordinary differential systems subject to linear constraints. SIAM J. Numer. Anal. 6 (1969), 8-30.

[6] Lucas, T., Asymptotic expansions for interpolating periodic splines, SIAM J. Numer. Anal., 19 (1982), 1051-1066.

[7] Lentini, M. and Pereyra, V., An adaptive finite difference solver for nonlinear twopoint boundary problems with mild boundary layers, SIAM J. Numer. Anal., 14 (1977), 91-111.

[8] Russell, R. and Christansen, J., Adaptive mesh selection strategies for solving boundary value problems, SIAM J. Numer. Anal., 15 (1978), 59-80.

[9] Russell, R. and Shampine, L., A collocation method for boundary value problems, Numer. Math., 19 (1972), 1-28.

[10] Sakai, M., Piecewise cubic interpolation and two-point boundary value problems, Publ. RIMS. Kyoto Univ. 7 (1972), 345-362.

[11] Shampine, L., Boundary value problems for ordinary differential equations. II. Patch bases and monotone methods, SIAM J. Numer. Anal. 6 (1969), 414-431.

[12] Scott, M., On the convergence of boundary value problems via several invariant imbedding algorithm, Numerical solutions of boundary value problems for ordinary differential equations, A. Aziz, ed., Academic Press, New York, 1974.

[13] Stepleman, R., Tridiagonally fourth order approximations to general two-point nonlinear boundary value problems with mixed boundary conditions, Math. Comp. 30 (1976), 92-103.

[14] Stetter, H., Asymptotic expansions for the error discretization algorithm for nonlinear functional equations, Numer. Math. 7 (1965), 18-31. 
